\title{
Creation and Control of Interactive Virtual Environments
}

\author{
Adrian Haffegee, Priscilla Ramsamy, Ronan Jamieson, and Vassil Alexandrov \\ Advanced Computing and Emerging Technologies Centre, \\ The School of Systems Engineering, University of Reading, \\ Reading, RG6 6AY, United Kingdom \\ sir04amh@reading.ac.uk
}

\begin{abstract}
Within the confines of a Virtual Environment (VE) almost anything is possible. It is easy to establish the benefits such an application could provide throughout the many walks of life, and yet current VE development remains within the domain of Virtual Reality application programmers. We describe methods that enhance VE development, first by providing scene creation for non-programmers, and second through a scene management entity that controls interaction within the environment. We explore methods for interacting through the scene to enable multiuser collaboration, and detail sample applications making use of this approach.
\end{abstract}

\section{Introduction}

The field of Virtual Reality (VR) concerns itself with the study of how the senses and perception can by fooled into believing that virtual objects exist, or that a non existing experience has occurred. This ability to alter what each of the senses is telling the brain, combined with the way the brain is translating them, provides vast scope in exploring the different aspects of reality. The uses of a perfect VR system are only limited by the imagination provided in development of its applications. Those depicting objects could have commercial uses in previewing or analyzing products. Alternatively medical based objects could provide surgeons a preview of what they will be facing without the prior need of investigative surgery. Indeed a virtual representation of any object could be created and then limitlessly studied and modified, thereby negating a large dependence on real world models or prototypes.

Rather than just depicting individual objects, an entire Virtual Environment (VE) could also be constructed. Representations of real environments could be used to experience products or locations, such as a new house or a holiday destination, while viewing from a different location.

Adding user interaction creates a more dynamic environment that could react to certain user provided stimulii. Examples here would include training exercises where users could be repeatedly placed inside simulations of otherwise difficult to reproduce situations, thereby learning as though they were in the real environment. However VEs need not just be constrained to environments that 
humans would naturally find themselves in. For instance animal behavior biologists may benefit from experiencing an environment as though they were seeing through the eyes of a different creature, or for entertainment purposes a user could become a hero in some fictional fantasy world.

The natural extension to having one person using a VE is to allow multiple users to share the environment. Ideally this should be possible regardless of the users' physical location, allowing geographically dispersed users to meet together in a Networked Virtual Environment (NVE) 1 ].

Once multiple users are connected together within a VE it is possible to use such an environment to replace current forms of remote user to user communication and interaction. These current methods make use of limited resources such as telephones, videoconferencing systems through to simple pens and paper. All of this functionality can brought into the almost limitless domain of the VE, making it a perfect medium supporting inter-person communications. However such technology extends beyond the boundaries imposed through the conventional resources; rather than just conveying their views to distant participants, users can interact with each other within their shared virtual world, dynamically manipulating it to convey their thoughts.

One issue effecting the usage of VEs is the effort taken in their development. While tools for their creation do exist [2, 3] they require programming experience along with a detailed knowledge of the area. This coupled with limited accessibility to VR hardware has resulted in restricted VE development. Current environments have little interaction between their users, with them often only sharing head and hand positioning and simple action events. Although some work has been undertaken in bringing realistically mixed audio into the environment [4, 5], generally external audio applications [6, 7] are used by VE participants.

Recently, the AGJuggler project [8] has attempted to bring the AccessGrid 9] conferencing technology into the virtual world. This is still a work in progress, and beyond the inclusion of remote video streams into the VE, currently has limited user interaction. However it does demonstrate areas where real world communications technology is beginning to merge into the VE.

Assuming the complexities of VE implementation can be ignored there also exists the problem of designing the environment itself. Whilst almost anyone could populate a blank environment with walls, floors, windows and doors, it would still take someone with architectural knowledge to be able to construct a practical and realistic virtual building. Similarly a psychologist would be needed to develop environments for psychological testing, or a doctor for simulating medical procedures. It is clear that the development of these advanced environments require skills that are not usually available for VR programmers.

This paper describes a method for creating and controlling interactive virtual environments that can be used by most people regardless of their computer programming background. Section 2 details the tools that achieve this functionality before section 3 describes how they can be used for different types of interaction. Section 4 describes sample applications built around this methodology and then the paper concludes with section 5 . 


\section{Controlling the Virtual World}

This work builds on top of a lower level VR application development toolset [10, that described a library of infrastructure tools providing functionality such as display management and user representation/control. A key development from this work was the creation of a common architectural platform for writing VR applications. Since they share the same source code, these applications could be used for cross system development from desktop PCs through to CAVEs [11.

The new additions presented here consist of a configuration tool that provides non-programmers with a method of constructing a VE from various virtual objects, and the scene controller which is a management entity that administers dynamic aspects of a scene.

\subsection{Scene Components}

Generally speaking immersive VR can be used to represent 2 different types of data; visualization and realistic, lifelike scenes. Visualization concerns itself with the visual representation and manipulation of data sets. It does not try to persuade the user that the data is anything more than what it is, and immersion is only used to provide improved representation. Lifelike scenes however are used to persuade the user that they really are in an alternate place. The greater the user feels attached to the scene and has a presence within it, the more successful the VE and the greater its likelihood of achieving its desired results. This split does not mean visualization cannot be used in lifelike scenes; on the contrary data sets are often used in such scenes, however then the visualization object is just a part of the full scene.

Similarly to the number of objects that surround us in real life, a virtual scene consists of a heterogeneous array of different virtual objects. Creating a believable lifelike scene depends on incorporating the right objects for the VE being built.

Objects created from primitive geometries have basic shapes such as spheres, boxes and cylinders. They are easy to define but lack the complexity required for many scenes. However they often have uses representing basic man-made objects such as balls, walls, tubes and so on.

More complex complex objects can be created through CAD or modeling packages. Assuming they are available in, or can be converted to an acceptable format, they can be brought into the VE as realistic representations. For example, an architect could create a CAD model of a house he is designing and add it life size to an environment. He could then enter the environment and examine or demonstrate his design before any materials have even been ordered, allowing potential modifications if required.

Textures can be added to objects to improve realism, and range from simple patterns to high quality images. Such textures can greatly enhance the virtual objects, for instance a basic box can be covered in a texture of a previously photographed item to create a realistic virtual representation (see figure 1). Being image based, textures can be enhanced for providing additional effects 

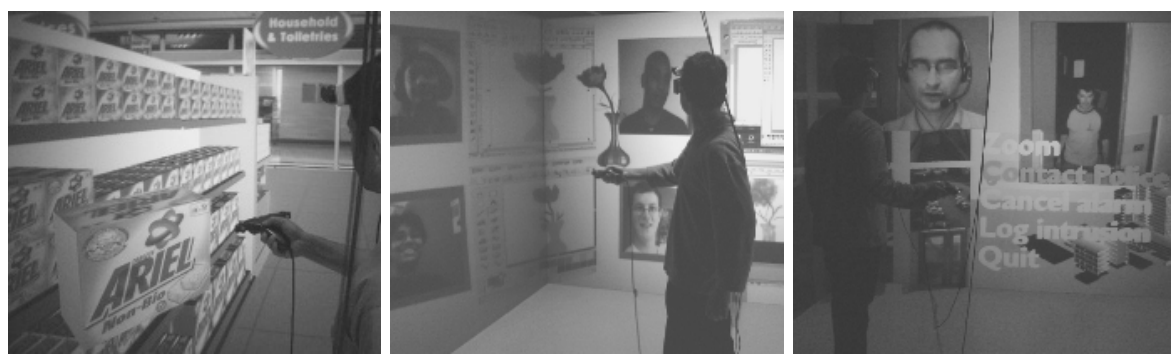

Fig. 1. CAVE applications demonstrating virtual shopping (left), multimedia conferencing (middle) and a virtual control room (right)

such as areas of transparency, lighting artifacts (reflections/shadows), aging or fogging. Textures do not need to remain static, and useful effects such as the illusion of passing time can be created from a dynamic sequences of different images.

While static objects are sufficient in a number of cases, it is often desirable for some degree of animation. In its simplest form objects can be made to move, either independently or resulting from user interaction. More advanced transformations could include scaling changes or morphing between objects.

\subsection{Scene Configuration}

The scope for potential VE development areas is vast. Its successful implementation would open huge avenues in many different fields. For instance it would become the perfect blank canvas for psychologists, artists and architects to name but a few. Using it they could create their environments tailored to their own specifications. However, these people generally do not possess the necessary VR programming skills to directly access the scene creation libraries. Simplified VR scripting languages (e.g VRML [12]) do exist, although being originally developed for creating/browsing 3D content on the Internet, have limited functionality in an immersive environment and lack widespread support for the various VR platforms. In an attempt to address the ease of creation issue, the Scene Configurator tool has been developed.

The Scene Configurator is a utility that creates a VE following some simple commands from a human readable configuration file. These commands define the objects that exist within the scene (geometries, models and any textural information), along with their location, animation and interaction characteristics. Using this approach non-programmers can create and edit the configuration files that manage their scenes. Currently proprietary plain text is used for the simplification of the commands, however it is anticipated that an additional text formating such as XML may be considered.

Having a common format for scene configuration enables further development of scene generation tools, enabling applications to store the state of their VE for backup or scene editing purposes. It also allows for automation in generating 
the scenes from from external data sources such as architectural design plans, shop-floor layouts and so on.

\section{$2.3 \quad$ Scene control}

An optional scene controller module has been added to the library of tools to free the application developer from some of the maintenance tasks within an environment. When used it creates and manages a registered hierarchy of virtual objects within the scene and can be used to provide animation and interaction. In addition to their geometries the objects have specific personalities effecting their behavior and enabling them to interact. Predefined personalities exist for these objects such as those that are manipulable (can attach to and be moved by a user or another object), mobile (they have their own rules determining how they move locally or around the scene) or are container objects (which can be used to hold/carry other objects). This base level of scene control can be provided to objects defined through the scene configurator. Additionally, further objects with extended characteristics and behavior can be derived from these base personalities.

Due to the underlying nature of the OpenSG scenegraph [13] that this work is built on, the object hierarchy has good support over distributed or multiprocessor architectures. This coupled with state storage in persistent or shared memory allows for more complex objects, or those that have additional processing requirements to exist without hampering rendering performance in the scene. An simple example of this could involve the dynamic motion and interaction of objects exposed to multiple forces. The scene controller may only need positional information from the objects, and this could be retrieved with minimal overhead. However additional threads (and processors) could be constantly calculating the physics behind the objects' motion to determine the resultant positions.

\section{User Interactions Within the Environment}

An extension of a single user interactive environment would involve multiple users, using aspects of the environment as a communication medium. Current applications sometimes use avatars [14,15, as virtual world representations of remote users as they share a VE. Here tracking devices follow user actions and map these onto their avatar counterpart. Using this method remote users can be seen to gesture or otherwise interact with the virtual world. However the expense of current tracking technology tends to result in a maximum of 2-3 tracked points for each person, thereby limiting its effectiveness.

A more acceptable method for interaction, and one that would help drive the uptake of VEs would be one that uses readily available technologies alongside the virtual world. Currently, most media formats revert to a $2 \mathrm{D}$ image such as those seen in books, televisions or displayed on PCs. While in the future it would be desirable for all users to be able to share and interact with 3D objects and models, right now these existing $2 \mathrm{D}$ formats can be incorporated into the $3 \mathrm{D}$ 
world. An example of this could be a virtual television or screen upon which could be displayed a traditional 2D image.

To facilitate this a particular type of virtual object has been developed with changeable textures each of which can represent a single frame/page of 2D data. This texture is read as needed from a shared memory segment attached to the object. The shared memory is written to by an external process based around the Multicast Application Sharing Tool (MAST) [16], which receives and decodes streams of application display data generated during group collaborations. More recently this tool has also been used for streaming face video. The resulting virtual object is therefore able to display a flat plane within the environment upon which can displayed live streams of video or application data. Since MAST supports multiple channels such an object could easily be used to represent a virtual monitor that could switch its display from one of many sources. Several of these could then be combined in the VE to form the basis of a multiple user multimedia conferencing system.

\section{Applications}

Several applications have already been developed from the described technologies, and demonstrate just some of the possible uses of these utilities. Figure 1 shows three of these applications running in a CAVE like environment.

The first of these is a virtual supermarket which allow users to pick up and examine different products. The whole scene has been developed with the scene configurator, so could easily have been created by a non-programmer. The store, the shelves and all the items on them as well as how they can be manipulated have been defined within the configuration file. The products and signs are simple geometries upon which textures created from photographs of the real objects have been added. Similar environments could just as easily be created to demonstrate alternative scenes, maybe museums containing artifacts that users could examine, or architectural designs to prototype new houses.

The second diagram shows a potential use of multimedia conferencing, in this case a virtual art class. MAST has been used to distribute application and face video from number of users. The teacher within the VE can view the students progress as they develop their drawing regardless of the application that each of them is using. The video and audio streams are provided to enhance communication between participants. This type of interaction could be used in many types of virtual meetings where audio, video and application data may wish to be shared amongst distributed users.

The final application shows a virtual environment being used as a security centred virtual control room. Multiple video streams from security cameras would be fed into the VE, and remote sensors could also be used to trigger alarm notification. The user from within the VE could be made aware of any intrusion, and enhance the respective video stream for further examination. Should it be necessary the user could notify the authorities while also having access to virtual models of the real environment to assist with any remedial action. Since it is 
unlikely that a user will be permanently required to monitor the same location, a virtual control centre could be established where a user could monitor many sites, each with their own virtual representation. If any of these sites require servicing then that VE could be brought up for the user's attention.

\section{Conclusion}

This work describes extensions to the VE development process that first open up the field to non-VR experts, and second provide a control entity that help in the management of dynamic virtual worlds. It discusses how virtual objects can be derived alongside these extensions to populate the environment with interactive items, and how these items can be used for multiuser collaboration and interaction. Finally it demonstrates applications that make use of this technology and suggests how they could be further enhanced.

Future work should extend the range of behaviors of the virtual objects and provide support for these within the scene configurator. Additional scene generation tools could provide automated or assisted development, potentially allowing extensive scene editing from within the VE. Improved collaborative interaction could also be developed through closer integration with MAST, potentially allowing bidirectional messaging for remote application communication and control.

\section{References}

1. Macedonia, M.R., Zyda, M.J.: A taxonomy for networked virtual environments. IEEE MultiMedia 4 (1997) 48-56

2. Rohlf, J., Helman, J.: IRIS performer: A high performance multiprocessing toolkit for real-time 3d graphics. In: SIGGRAPH, ACM Press (1994) 381-394

3. Bierbaum, A.D.: VR Juggler: A virtual platform for virtual reality application development. Master's thesis, Iowa State University, Ames, Iowa (2000)

4. Radenkovic, M., Greenhalgh, C., Benford, S.: A scaleable audio service for CVEs. In: Proc of the sixth conference of the UK VRSIG. (1999)

5. Neumann, T., Fünfzig, C., Fellner, D.W.: TRIPS - a scalable spatial sound library for OpenSG. Technical Report TUBSCG-2003-02, Institute of ComputerGraphics (2003)

6. Robust Audio Tool (RAT) website. Available on: http://www-mice.cs.ucl.ac. uk/multimedia/software/rat

7. Teamspeak website. Available on: http://www.goteamspeak.com

8. Gonzalez, D.: AGJuggler: An architecture for virtual reality within a collaboration environment. Master's thesis, Purdue University (2005)

9. Childers, L., Disz, T., Olson, R., Papka, M., Stevens, R., Udeshi, T.: Access grid: Immersive group-to-group collaborative visualization. In: 4th International Immersive Projection Technology Workshop. (2000)

10. Haffegee, A., Jamieson, R., Anthes, C., Alexandrov, V.: Tools for collaborative VR application development. In: International Conference on Computational Science, Springer Verlag (2005) 350-358

11. Cruz-Neira, C., Sandin, D.J., Defanti, T.A., Kenyon, R.V., Hart, J.C.: The CAVE: Audio visual experience automatic virtual environment. Communications of the ACM 35 (1992) 64-72 
12. Carey, R., Bell, G.: The VRML 2.0 annotated reference manual. Addison-Wesley, Reading, MA, USA (1997)

13. Reiners, D.: OpenSG: A Scene Graph System for Flexible and Efficient Realtime Rendering for Virtual and Augmented Reality Applications. PhD thesis, Technische Universität Darmstadt (2002)

14. Badler, N.I., Phillips, C.B., Webber, B.L.: Simulating Humans: Computer Graphics Animation and Control. Oxford University Press, New York, NY, USA (1992)

15. Park, K., Cho, Y., Krishnaprasad, N., Scharver, C., Lewis, M., Leigh, J., Johnson, A.: CAVERNsoft G2: A toolkit for high performance tele-immersive collaboration. In: VRST, Seoul, Korea, ACM Press (2000) 8-15

16. Lewis, G.J., Hassan, S.M., Alexandrov, V.N., Dove, M.T., Calleja, M.: Multicast application sharing tool - facilitating the eminerals virtual organization. In: International Conference on Computational Science, Springer Verlag (2005) 359-366 